\title{
Declaración de Cancún: declaración internacional de Cancún sobre el derecho a la nutrición en los hospitales
}

\author{
Cancun Declaration: international declaration of Cancún \\ on the right to nutrition in hospitals
}

\author{
J. C. de la Cruz Castillo Pineda', R. Figueredo Grijalva ${ }^{2}$, C. Dugloszewski ${ }^{3}$, \\ J. A. S. Ruy Díaz Reynoso ${ }^{4}$, J. V. Spolidoro Noroña ${ }^{5}$, A. Matos ${ }^{6}$, F. Carrasco ${ }^{7}$, G. Chirife \\ A. Vergara ${ }^{9}$, J. Moya Rodríguez ${ }^{10}$, G. Loyola ${ }^{11}$, A. Álvarez ${ }^{12}$, C. Barozzi ${ }^{13}$, M. Vargas ${ }^{14}$ \\ y A. García de Lorenzo ${ }^{15}$.
}

Recibido: 1 de julio de 2018. Aceptado para publicación: 15 de agosto de 2018 https://doi.org/10.35454/rncm.v1n2.047

La declaración de Cancún por el derecho humano de los enfermos a recibir una terapia nutricional oportuna y óptima en cualquier lugar donde se encuentren ha sido emitida por los Presidentes de las Sociedades y Asociaciones de Nutrición Clínica y Terapia Nutricional de América Latina y el Caribe, en el marco del XI Congreso de FELANPE/XV Congreso de AMAEE, en la Ciudad de Cancún Quintana Roo, México, el día 20 de mayo de 2008.

\section{PREÁMBULO}

Considerando que la libertad, la justicia y la paz en el mundo tienen por base el reconocimiento de la

1 Presidente de la Federación Latinoamericana de Terapia Nutricional Clínica y Metabolismo 2008.

2 Sociedad Uruguaya de Nutrición (SUN).

3 Asociación Argentina de Nutrición Enteral y Parenteral (AANEP).

Asociación Mexicana de Nutrición Clínica y Terapia Nutricional (AMAFE).

Sociedad Brasileña de Nutrición Parenteral y Enteral (SBNPE).

6 Sociedad Panameña de Nutrición Clinica (SPNC).

7 Asociación Chilena de Nutrición Clínica y Metabolismo (ACNCM).

8 Asociación Paraguaya de Nutrición (APN).

9 Asociación Colombiana de Nutrición Clínica (ACNC).

10 Asociación Costarricense de Nutrición Parenteral y Enteral (ACONEP). dignidad intrínseca y de los derechos iguales e inalienables de todos los miembros de la familia humana; Considerando que los pueblos de las Naciones Unidas han reafirmado en la Carta su fe en los derechos fundamentales del hombre, en la dignidad y el valor de la persona humana y en la igualdad de derechos de hombres y mujeres, y se han declarado resueltos a promover el progreso social y a elevar el nivel de vida dentro de un concepto más amplio de la libertad; Considerando que las Asociaciones y Sociedades Latinoamericanas afiliadas a la Federación Latinoamericana de Terapia Nutricional, Nutrición Clínica y Metabolismo (FELANPE) se han comprometido a asegurar, el res-

\footnotetext{
11 Asociación Salvadoreña de Nutrición Parenteral y Enteral (ASNEP).

12 Sociedad Cubana de Nutrición Clinica (SCNC).

13 Sociedad Uruguaya de Nutrición (SUN).

14 Sociedad Venezolana de Nutrición Parenteral y Enteral (SVNPE).

15 Sociedad Española de Nutrición Parenteral y Enteral (SENPE).

Publicado en (2008):

Castillo Pineda JC, Figueredo Grijalva R, Dugloszewski C, Ruy Díaz Reynoso JAS, Spolidoro Noroña JV, Matos A, et al. Declaración de Cancún: declaración internacional de Cancún sobre el derecho a la nutrición en los Hospitales. Nutr Hosp. 2008;23(5):413-7.
} 
peto universal y efectivo a los derechos y libertades fundamentales del hombre contenidos en la Declaración Universal de los Derechos Humanos adoptada y proclamada por la resolución de la Asamblea General 217 (iii) el 10 de diciembre de 1948, Considerando que La FELANPE tiene como finalidad el congregar asociaciones o sociedades médicas, de enfermería, farmacéuticas, nutricionistas y demás profesionales del área de la salud, que ejerzan la especialidad de la Terapia Nutricional y Metabolismo, para incentivar y estimular el estudio, pesquisas, educación continuada y formación de profesionales en Nutrición Clínica, y colaborar si así se requiriera con el Poder Público de los países miembros y entidades vinculadas a los asuntos de Salud, en la evaluación y solución de los problemas relativos a la Nutrición Clínica, mantener intercambios técnicos, científicos y asociativos con entidades congéneres internacionales, Velar por la ética y calidad técnica del ejercicio profesional de la Terapia nutricional entre otros, Considerando que el problema de la desnutrición hospitalaria en Latinoamérica es un problema grave como lo ha puesto en evidencia múltiples investigaciones a lo largo del mundo, destacando el estudio multicéntrico ELAN publicado en 2003 donde resalta que la malnutrición está presente en más de la mitad de los pacientes hospitalizados y que era poca la información en el expedientes y sobre todo que solo una mínima parte de ellos recibían terapia nutricional y que solo dos países en Latinoamérica (Brasil y Costa Rica) tienen una política gubernamental que norma la práctica de la terapia nutricional.

\section{Por tanto}

1. Nosotros, los Presidentes de la Sociedades y Asociaciones de Nutrición Clínica y Terapia Nutricional afiliados a la Federación Latinoamericana de Terapia Nutricional, Nutrición Clínica y Metabolismo (FELAPE), de Chile, Argentina, Uruguay, Paraguay, Brasil, Perú, Ecuador, Colombia, Venezuela, Panamá, Costa Rica, El Salvador, Honduras, Guatemala, México, Cuba, Puerto Rico, República Dominicana y España; así como solidarios la Asociación Americana de Nutrición Parenteral y Enteral (ASPEN) y la Sociedad Europea de Nutrición Clínica y Metabolismo (ESPE), reunidos en la ciudad de Cancún, Quintana Roo, México con motivo del XI Congreso Latinoamericano del 18 al 21 de mayo de 2008, declaramos que es nuestro deseo y compromiso comunes para garantizar que todo enfermo sea valorado nutricionalmente al ingreso a los servicios de salud de primero, segundo y tercer nivel de atención del sector público, privado o de asistencia social del mundo y en especial de Latinoamérica y reciba una atención nutricional oportuna, completa, suficiente y de calidad, proporcionada por profesionales de la Salud capacitados, que prevenga el riesgo de la desnutrición hospitalaria y reduzca el número y la severidad de las complicaciones relacionadas con el proceso de la enfermedad que llevó al paciente a hospitalizarse, además de mejorar su Calidad de Vida, su sobrevida y reducir los costos relacionados con la atención de la enfermedad en estos hospitales. Así también a los enfermos por exceso y/o desbalance en su alimentación o por alteraciones en su metabolismo cursan con sobrepeso y obesidad dado que en la actualidad se considera una verdadera epidemia que afecta a toda la población incrementando las complicaciones médicas, aumentando los ingresos y las complicaciones hospitalarias, la estancia en el hospital y que requiere tanta atención como el desnutrido.

2. Nuestro desafío es promover y garantizar como líderes de opinión en nuestros respectivos países el derecho humano de los enfermos a recibir una terapia nutricional oportuna y óptima en cualquier lugar donde se encuentren.

3. Reafirmamos la universalidad, indivisibilidad, interdependencia e interrelación de todos los derechos humanos y las libertades fundamentales, incluido el derecho a la alimentación, tal como se consagra en la Declaración Universal de los Derechos humanos.

4. Reafirmamos nuestro compromiso con lo dispuesto en el Artículo 25 de la Declaración Universal de Derechos Humanos, a saber, toda persona tiene derecho a un nivel de vida adecuado que le asegure, así como a su familia, la salud y el bienestar, y en especial la alimentación, el vestido, la vivienda, la asistencia médica y los servicios sociales necesarios; tiene asimismo derecho a los seguros en caso de desempleo, enfermedad, invalidez, viudez, vejez u otros casos de pérdida de sus medios de subsistencia por circunstancias independientes de su voluntad. Estos derechos y libertades no podrán en ningún caso ser ejercidos en oposición a los propósitos y principios de las Naciones Unidas. De esta manera, fomentaremos una Sociedad en la que se respete la dignidad de la persona humana.

5. Reconocemos que la ciencia desempeña un papel cardinal en el desarrollo de la Sociedad. Gran parte de los elementos constitutivos de las sociedades son el fruto de los avances científicos y técnicos que han sido posibles gracias a la comunicación mutua de los resultados de la investigación. 
6. Somos plenamente conscientes que la malnutrición es una entidad compleja y multifactorial, pero cualquiera que sean las causas, sus consecuencias clínicas pueden ser muy serias, conduciendo a complicaciones de una enfermedad, pobre respuesta a la terapia medicamentosa, disminución de la inmunocompetencia y aumento de la Morbi-mortalidad. Mientras que las consecuencias económicas incluyen el aumento en el costo de la estancia hospitalaria y tratamientos adicionales, creando un impacto muy serio en los sistemas de salud.

7. Afirmamos que las Sociedades y Asociaciones de profesionales de la Salud de América Latina interesadas en el estudio, la difusión, la investigación y la educación relacionada con la nutrición clínica y la terapia nutricional deben cerrar filas en torno a este serio problema de salud que aqueja a una buena parte de los pacientes ingresados en hospitales del sector público, privado y social de los diferentes países que conforman la región.

8. Somos conscientes que uniendo esfuerzos se presentará un frente común y coordinado que permita que cualquier paciente ingresado en el hospital tenga acceso a un Derecho fundamental de la Persona $\mathrm{Hu}$ mana como lo es la Nutrición oportuna, completa, suficiente y de calidad durante su internamiento y sobre todo a la prevención de la desnutrición hospitalaria a través de procesos hospitalarios bien estructurados.

9. Nada en la presente Declaración podrá interpretarse o derogue las disposiciones de la Carta de las Naciones Unidas y la Declaración Universal de Derechos Humanos, así como de ningún otro instrumento internacional o de las leyes nacionales adoptadas de conformidad con esos instrumentos.

10.Acordamos que nuestros esfuerzos y compromisos deberán encausarse en la siguientes líneas de acción, a saber:

1. Evaluación y Tratamiento Nutricionales en los Hospitales:

a. La atención nutricional no es una opción, es una necesidad impostergable para todo paciente. El soporte nutricional es obligatorio para todo paciente malnutrido o en riesgo. La finalidad del mismo es brindar y satisfacer las necesidades del organismo, aportando los nutrientes precisos cuantitativa y cualitativamente en forma oportuna.

b. Todo paciente hospitalizado debe ser evaluado dentro de las primeras 24 a 48 horas, con un método de tamizaje validado, y de acuerdo a su resultado deberá ser derivado para evaluación nutricional formal.

c. El cuidado nutricional del paciente hospitalizado y la administración del soporte nutricional requieren de la implementación de guías de práctica clínica que contemplen:

i. El método de tamizaje nutricional en todos los hospitales.

ii. Evaluación metabólica-nutricional formal. iii. Implementación del plan de alimentación y/o terapia nutricional.

iv. Programa de seguimiento.

v. Reevaluación y reformulación del plan y/o terapia o su finalización.

d. El soporte nutricional es más costo-efectivo cuando es realizado por un grupo especializado. Cada institución debe contar con un grupo interdisciplinario responsable del Soporte $\mathrm{Nu}$ tricional. Si esto no es posible cada institución debe proveer la mejor estructura responsable de la realización adecuada del Soporte Nutricional.

e. Las sociedades científicas deben proveer capacitación y actualización; así como definir guías de práctica clínica. Los sistemas nacionales de salud tienen que sostener y regular su aplicación, todo esto para que:

i. Los pacientes tengan garantizado su derecho a recibir el óptimo tratamiento alimentario-nutricional independientemente del lugar donde se encuentren.

ii. Los trabajadores de la salud estén respaldados en su práctica clínica.

iii.Los sistemas nacionales de salud realicen la vigilancia epidemiológica, fiscalización y planificación financiera.

2. Proveedores profesionales de los Cuidados Nutricionales:

a. En vista de la importancia que reviste el cuidado nutricional en nuestros hospitales, Se insta de manera categórica a los encargados de dirigir estos centros, a promover de manera responsable, la constitución de Equipos de Terapia Nutricional acreditados en cada uno de ellos, y a dar la correcta consecución a estos equipos a fin de garantizarla un nivel óptimo de atención a nuestros pacientes.

b. Se insta a las diferentes escuelas de Ciencias de la Salud, a incluir en el Currículum de Estudios, la materia Nutrición Clínica en el Pensum 
Académico de Pre-grado y Postgrado, a fin que los estudiantes reciban una sólida formación en la materia, y sean en un futuro portavoces del cuidado nutricional de los pacientes.

c. Se recomienda, a las diferentes Instituciones de Salud, tomar en cuenta las normas de las Organizaciones Internacionales Reguladoras, en cuanto al número adecuado de Nutricionistas que deberían laborar en los diferentes Centros Asistenciales.

d. Tomando en cuenta la disminución de costos que significa, el buen cuidado del estado nutricional al evitar las complicaciones, Los Administradores de Salud, como norma indiscutible deben garantizar, exigir y facilitar la Educación continua del personal a cargo en la materia.

e. Es responsabilidad del servicio de enfermería, llevar un registro de consumo alimentario y tomar las acciones necesarias cuando se detecte la disminución en la ingesta.

3. Prácticas de los Servicios Hospitalarios de Alimentos:

a. Organización del Servicio Hospitalario de Alimentos.

i. Legislaciones a nivel nacional que rijan la implementación de los servicios de alimentación hospitalaria.

ii. Políticas y planes hospitalarios estratégicos tendientes a fortalecer el Servicio de Alimentación de nuestros Hospitales.

iii. Servicios de Alimentación dirigidos por Profesionales capacitados en Nutrición Humana y en Gerencia de recursos humanos.

b. Menús de alimentos y dietas médicamente prescritas.

i. Manual de dietas, que incluya los objetivos de cada una de ellas y sus especificaciones técnicas (condiciones organolépticas, horarios, temperatura, acordes con las necesidades, gustos, preferencias, costumbres y cultura de los pacientes). Las mismas deben estar a disposición de los profesionales del Servicio del Alimentación y de todo el personal del Hospital.

ii. Equipos multidisciplinarios que realicen capacitaciones permanentes sobre dicho Manual.

4. Nutrición Pediátrica:

a. Evaluación Nutricional. Debe garantizar una herramienta que permita identificar el riesgo nutricional de todo paciente pediátrico que ingrese en un hospital.

b. Indicaciones de apoyo nutricional. El apoyo nutricional debe indicarse en todo paciente pediátrico que lo requiera de acuerdo a su patología de base, cuando los requerimientos no puedan ser cubiertos mediante una dieta convencional, deberá administrarse por la vía de acceso enteral o parenteral según su condición patológica.

c. Terapéutica nutricional. Todo paciente hospitalizado deberá contar desde su ingreso con un plan de cuidado nutricional acorde a la evaluación arriba mencionada, que debe ser reevaluado y ajustado a la evolución del paciente. De igual forma debe garantizarse la disponibilidad de leche materna y en casos necesarios de fórmulas infantil o especializadas por patología.

En caso de no poder utilizar el tracto gastrointestinal, se debe garantizar la existencia de soluciones de nutrientes para empleo parenteral adaptadas a la edad pediátrica, y favorecer la implementación de centros de preparación de mezclas que garanticen la óptima calidad.

d. Aspectos Bioéticos. Se debe asegurar que se cumplan los principios universales de bioética: justicia, no maleficencia, autonomía y beneficencia.

e. Aspectos legales. Deberán de registrarse en el expediente clínico: la evaluación nutricional, el diagnóstico y el plan de cuidado o tratamiento nutricional. Las fórmulas o mezclas nutricionales para uso enteral y parenteral deben cumplir con los requerimientos de identificación y rotulación.

5. Economía de la Salud:

a. Considerando que:

i. Es factible que la FELANPE, a través de sus agremiados, promueva iniciativas que culminen en el reforzamiento de medidas legislativas, reglamentos oficiales, normas técnicas y guías clínicas, influyendo en los sistemas de salud latinoamericanos,

ii. Es factible que la FELANPE, a través de sus enlaces inter e intra institucionales, académicos y universitarios, favorezca un entorno en que los pacientes hospitalizados se empoderen de nuevos derechos a favor de su bienestar,

iii. El grupo responsable de emitir recomendaciones, en materia de economía de la salud, 
referentes a la Declaración sobre el derecho a la Nutrición en los Hospitales de Latinoamérica, estableció los siguientes.

b. Subtemas:

i. Sobre los sistemas de salud latinoamericano (Conclusiones 1, 2 y 3).

ii. Sobre los pacientes hospitalizados (Conclusiones 4,5 y 6 ).

Una vez analizados según la metodología establecida por la FELANPE, bajo las siguientes:

c. Definiciones.

i. Los Sistemas de salud latinoamericanos pueden alcanzar el nivel de convertir la nutrición artificial en un derecho para los pacientes hospitalizados.

ii. Una atención nutricional de calidad incluye la seguridad, oportunidad, eficiencia, eficacia, efectividad, equidad y sin modificar los valores bioéticos.

iii. La economía en salud se basa en indicadores de:

- Costo minimización, que reduce los costos a favor de las utilidades.

- Costo eficacia, que favorece la atención médica a menor costo.

- Costo beneficio, que mejora las utilidades independientemente del costo.

- Costo efectividad, que favorece acciones nuevas a menor costo.

- Costo utilidad, que incrementa los años de vida potencialmente saludables.

d. Conclusiones:

i. Los sistemas de salud latinoamericanos deben establecer la normatividad y los procesos que garanticen que los pacientes hospitalizados reciban una atención nutricional de calidad.

ii. Los sistemas de salud latinoamericanos, al cumplir con una nueva normatividad en materia de nutrición hospitalaria, deberán implementar las estrategias basadas en la mejor evidencia y que les permitan una atención nutricional de calidad, así como medir el costo minimizado de las mismas.

iii.Los sistemas de salud latinoamericanos, garantizarán la educación y promoción necesaria para que los pacientes hospitalizados participen activamente en el cumplimiento del objetivo nutricional planteado entre él y su personal de salud, procurando que las acciones sean costo eficaces. iv. Los pacientes hospitalizados tienen derecho a un diagnóstico de riesgo nutricional y ser informados del costo beneficio de recibir una intervención nutricional de calidad.

v. Los pacientes hospitalizados tienen derecho a recibir una atención nutricional de calidad, con personal calificado en el tema y procurando costo efectividad.

vi. Los pacientes hospitalizados tienen derecho a que se asignen recursos según las políticas públicas enfocadas a la promoción, prevención e intervención nutricional bajo esquemas de costo utilidad que resulten en una mejor calidad de vida.

Bajo las siguientes líneas de acción:

- Fomentar, a través de grupos de expertos, la elaboración de guías clínicas de comprobada eficacia, que permitan establecer protocolos unificados.

- Documentar y publicar las evidencias mundiales en terapia nutricional.

- Proponer a las autoridades las mejores prácticas educacionales para aplicar en los pacientes.

- Promocionar las evaluaciones que midan eficazmente los riesgos.

- Favorecer estudios de impacto económico en materia de terapia nutricional.

- Favorecer estudios de impacto económico en materia de terapia nutricional.

- Entablar mecanismos de contacto con autoridades legislativas para favorecer mejores presupuestos en esta materia.

6. Investigación en Nutrición Clínica:

a. Se requiere estimular la creación de oportunidades de educación, metodología en investigación y bioética en nutrición clínica.

b. Es importante investigar acerca de los recursos disponibles tanto materiales e intelectuales de cada región.

c. Identificar distintas líneas de investigación: epidemiológicas, clínicas, de ciencias básicas y de ciencias sociales.

d. Llevar a cabo un censo detallado de investigadores en Latinoamérica.

e. Promover un estudio epidemiológico de desnutrición hospitalaria esclareciendo que ha cambiado en los últimos 10 años después del estudio ELAN. 
La Firma de la Declaración se realizó con los siguientes testigos:

- Dr. Alberto Lifshitz. Representante del Presidente Constitucional de los Estados Unidos Mexicanos.

- Dr. Fernando Lolas Stepke. Comisión de Bioética de la Organización Panamericana de la Salud (OPS/ OMS).

- Dr. Ector Jaime Ramírez Barba. Presidente de la Comisión de Salud de la H. Cámara de Diputados de los Estados Unidos Mexicanos.

- Dr. José Antonio Carrasco Bojas. Presidente de la Academia Mexicana de Cirugía.
- Dr. Tomás Barrientos Fortes. Presidente de la Asociación Internacional de Facultades Católicas de Medicina de la Federación Internacional de Universidades Católicas.

- Dr. Antonio Crespo Burgos. Pontificia Universidad de Quito Ecuador.

- Dr. Olle Ljunjqvist. Asociación Europea de Nutrición Clínica y Metabolismo.

- Dr. Vincent Vanek. Sociedad Americana de Parenteral y Enteral.

- Dr. Gil Hardy. Vicepresidente de Sociedad Australiana de Nutrición Parenteral y Enteral. 